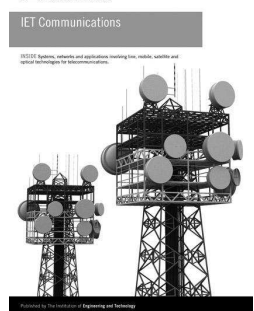

ISSN 1751-8628

\title{
Single-carrier frequency domain equalisation with hierarchical constellations: an efficient transmission technique for broadcast and multicast systems
}

\author{
J. Silva $a^{1,2}$ R. Dinis ${ }^{1,3,4}$ N. Souto ${ }^{1,2}$ P. Montezuma ${ }^{3,4}$ \\ ${ }^{1} I T$, Instituto de Telecomunicações, Av. Rovisco Pais, Lisboa, Portugal \\ ${ }^{2}$ ISCTE, Instituto Superior das Ciências do Trabalho e da Empresa, Av. das Forças Armadas, Lisboa, Portugal \\ ${ }^{3}$ DEE, Faculdade de Ciências e Tecnologia, FCT, Universidade Nova de Lisboa, 2829-516 Caparica, Portugal \\ ${ }^{4}$ Uninova, Instituto de D'esenvolvilmento de Novas Tecnologias, Quinta da Torre, Caparica, Portugal \\ E-mail: joao.silva@iscte.pt
}

\begin{abstract}
Orthogonal frequency division multiplexing (OFDM) schemes are the choice modulation for broadband wireless broadcast and multicast systems. However, OFDM schemes have important limitations such as high-envelope fluctuation of the transmitted signals and its sensibility to carrier frequency errors. When these limitations prove critical the authors consider single-carrier frequency-domain equalisation (SC-FDE) schemes, that allow much higher power efficiency because of lower envelope fluctuations. The overall performance can be further improved if the conventional linear FDE is replaced by an iterative FDE such as an iterative block decision feedback equaliser (IB-DFE). Conventional IB-DFE are usually designed for a quadrature phase shift keying constellation. However, it is strongly recommended that broadcast and multicast systems employ hierarchical constellations with several classes of bits with different error protection. In this study the authors consider the use of SC-FDE schemes combined with IB-DFE receivers in broadband wireless broadcast and multicast systems. The authors emphasise the advantages of these schemes and the authors present IB-DFE designs suitable for hierarchical constellations with several classes of bits with different error protection.
\end{abstract}

\section{Introduction}

Although originally broadcasting meant the sowing of seeds throughout a large field [1], as [2] it is generally associated with the distribution of any type of content to all users capable of receiving such information, for example the transmission of a TV signal to all users with a TV set. A derived term, multicast, is the distribution of content to a particular group of users, belonging to the multicast group [3] (e.g. users that paid for a given pay-per-view programme, or subscribed to a specific service).

The design of broadband wireless broadcast and multicast systems is a considerable challenge. In fact, broadband systems boast of high-bit rates, whose data are subject to severe time-dispersion effects because of multipath propagation effects. In addition, we need to transmit the same information to users that can have substantially different propagation conditions (e.g. users close to the transmitter and users at the edge of the cell). Multicast systems also have to consider that only users belonging to the group should receive the information and as such require specialised algorithms at higher layers [4-6].

Since orthogonal frequency division multiplexing (OFDM) schemes [7] are suitable for severely time-dispersive channels, they were the choice modulation for digital audio broadcasting (DAB) [8] and digital video broadcasting
(DVB) [9]. As we need to send the same information to users with substantially different propagation conditions (and, inherently, different signal-to-noise ratio (SNR) levels), OFDM schemes are usually combined with hierarchical constellations [10-12]. By allowing multiresolution schemes, different users will have different perceptions of the modulation according to their SNR conditions. The use of multi-resolution consists of sending a hierarchical constellation whose symbols are mapped in such a way that we have different error protection levels, which means that the distance between the symbols associated with each bit may vary from bit to bit.

However, OFDM schemes have some drawbacks. One of the most important drawbacks of OFDM schemes is the high envelope fluctuation and peak-to-average power ratio (PAPR) of the transmitted signals, that leads to amplification difficulties since we need amplifiers that are linear in a high range which usually means high-backoff and reduced amplification efficiency [13]. To reduce the required backoff we can employ clipping and filtering techniques [14-17]. However, this not only leads to performance degradation, which is more serious for the least protected bits of hierarchical constellations, but also the resulting envelope fluctuations are still larger than those with conventional single-carrier modulations based on the same constellations. Another drawback of OFDM schemes 